\title{
Di- and Tetranuclear Metal Complexes with Phenoxo Bridges, Synthesis, Structures, Photoluminescent and Electroluminescent Properties
}

Hong-Yu. Zhang, Kai-Qi. Ye, Jing-Ying. Zhang, Yin. Liu, Yue. Wang*

Key Laboratory for Supramolecular Structure and Materials of Ministry of Education, College of Chemistry, Jilin University, Changchun 130012, People's Republic of China

Corresponding author. Fax: +86-431-5193421. E-mail: yuewang@jlu.edu.cn. 


\section{S-Table 1.}

S-Table 1. Selected bond lengths $[\AA]$ and angles $\left[^{\circ}\right]$ for 2 . symmetry codes:-x-1,-y+1,-z.

\begin{tabular}{|c|c|c|c|}
\hline$\overline{\mathrm{Cu}(1)-\mathrm{O}(2)}$ & $1.900(4)$ & $\mathrm{O}(2)-\mathrm{Cu}(1) \# 1$ & $2.079(4)$ \\
\hline $\mathrm{Cu}(1)-\mathrm{O}(1)$ & $1.909(4)$ & $\mathrm{O}(3)-\mathrm{C}(18)$ & $1.351(7)$ \\
\hline $\mathrm{Cu}(1)-\mathrm{N}(1)$ & $2.014(5)$ & $\mathrm{O}(4)-\mathrm{C}(34)$ & $1.339(7)$ \\
\hline $\mathrm{Cu}(1)-\mathrm{O}(2) \# 1$ & $2.079(4)$ & $\mathrm{O}(5)-\mathrm{C}(37)$ & $0.952(14)$ \\
\hline $\mathrm{Cu}(1)-\mathrm{O}(3)$ & $2.195(4)$ & $\mathrm{N}(1)-\mathrm{C}(7)$ & $1.349(8)$ \\
\hline $\mathrm{Cu}(2)-\mathrm{O}(4)$ & $1.836(5)$ & $\mathrm{N}(1)-\mathrm{C}(11)$ & $1.357(8)$ \\
\hline $\mathrm{Cu}(2)-\mathrm{O}(3)$ & $1.901(4)$ & $N(2)-C(24)$ & $1.351(8)$ \\
\hline $\mathrm{Cu}(2)-\mathrm{N}(2)$ & $1.976(5)$ & $\mathrm{N}(2)-\mathrm{C}(28)$ & $1.370(7)$ \\
\hline $\mathrm{Cu}(2)-\mathrm{O}(1)$ & $1.984(4)$ & $\mathrm{N}(3)-\mathrm{C}(37)$ & $1.390(16)$ \\
\hline $\mathrm{O}(1)-\mathrm{C}(1)$ & $1.349(7)$ & $\mathrm{N}(3)-\mathrm{C}(35)$ & $1.399(14)$ \\
\hline $\mathrm{O}(2)-\mathrm{C}(17)$ & $1.338(7)$ & $\mathrm{N}(3)-\mathrm{C}(36)$ & $1.445(13)$ \\
\hline $\mathrm{O}(2)-\mathrm{Cu}(1)-\mathrm{O}(1)$ & $178.27(18)$ & $\mathrm{N}(2)-\mathrm{Cu}(2)-\mathrm{O}(1)$ & $161.05(18)$ \\
\hline $\mathrm{O}(2)-\mathrm{Cu}(1)-\mathrm{N}(1)$ & $91.0(2)$ & $\mathrm{C}(1)-\mathrm{O}(1)-\mathrm{Cu}(1)$ & $118.5(4)$ \\
\hline $\mathrm{O}(1)-\mathrm{Cu}(1)-\mathrm{N}(1)$ & $89.7(2)$ & $\mathrm{C}(1)-\mathrm{O}(1)-\mathrm{Cu}(2)$ & $134.5(4)$ \\
\hline $\mathrm{O}(2)-\mathrm{Cu}(1)-\mathrm{O}(2) \# 1$ & $78.0(2)$ & $\mathrm{Cu}(1)-\mathrm{O}(1)-\mathrm{Cu}(2)$ & $105.9(2)$ \\
\hline $\mathrm{O}(1)-\mathrm{Cu}(1)-\mathrm{O}(2) \# 1$ & $102.37(17)$ & $\mathrm{C}(17)-\mathrm{O}(2)-\mathrm{Cu}(1)$ & $115.9(4)$ \\
\hline $\mathrm{N}(1)-\mathrm{Cu}(1)-\mathrm{O}(2) \# 1$ & $141.74(17)$ & $\mathrm{C}(17)-\mathrm{O}(2)-\mathrm{Cu}(1) \# 1$ & $137.9(4)$ \\
\hline $\mathrm{O}(2)-\mathrm{Cu}(1)-\mathrm{O}(3)$ & $103.22(17)$ & $\mathrm{Cu}(1)-\mathrm{O}(2)-\mathrm{Cu}(1) \# 1$ & $102.0(2)$ \\
\hline $\mathrm{O}(1)-\mathrm{Cu}(1)-\mathrm{O}(3)$ & $75.06(16)$ & $\mathrm{C}(18)-\mathrm{O}(3)-\mathrm{Cu}(2)$ & 116.1(3) \\
\hline $\mathrm{N}(1)-\mathrm{Cu}(1)-\mathrm{O}(3)$ & $114.24(17)$ & $\mathrm{C}(18)-\mathrm{O}(3)-\mathrm{Cu}(1)$ & 134.1(4) \\
\hline $\mathrm{O}(2) \# 1-\mathrm{Cu}(1)-\mathrm{O}(3)$ & $103.97(15)$ & $\mathrm{Cu}(2)-\mathrm{O}(3)-\mathrm{Cu}(1)$ & $98.48(18)$ \\
\hline $\mathrm{O}(4)-\mathrm{Cu}(2)-\mathrm{O}(3)$ & $166.29(18)$ & $\mathrm{C}(34)-\mathrm{O}(4)-\mathrm{Cu}(2)$ & 118.1(4) \\
\hline $\mathrm{O}(4)-\mathrm{Cu}(2)-\mathrm{N}(2)$ & $92.0(2)$ & $\mathrm{C}(7)-\mathrm{N}(1)-\mathrm{Cu}(1)$ & $119.8(5)$ \\
\hline $\mathrm{O}(3)-\mathrm{Cu}(2)-\mathrm{N}(2)$ & $95.1(2)$ & $\mathrm{C}(11)-\mathrm{N}(1)-\mathrm{Cu}(1)$ & $118.8(5)$ \\
\hline $\mathrm{O}(4)-\mathrm{Cu}(2)-\mathrm{O}(1)$ & $96.41(18)$ & $\mathrm{C}(24)-\mathrm{N}(2)-\mathrm{Cu}(2)$ & $119.5(4)$ \\
\hline $\mathrm{O}(3)-\mathrm{Cu}(2)-\mathrm{O}(1)$ & $80.47(17)$ & $\mathrm{C}(28)-\mathrm{N}(2)-\mathrm{Cu}(2)$ & $119.3(4)$ \\
\hline
\end{tabular}




\section{S-Table 2.}

S-Table 2. Selected bond lengths $[\AA]$ and angles $\left[{ }^{\circ}\right]$ for 3 symmetry codes: \#1 -x+2,-y,-z+2, $\# 2-\mathrm{x}+1,-\mathrm{y}+1,-\mathrm{z}+1$.

\begin{tabular}{|c|c|c|c|}
\hline$\overline{\mathrm{Zn}(1)-\mathrm{O}(1)}$ & $1.989(5)$ & $\mathrm{Zn}(3)-\mathrm{O}(6)$ & $2.037(5)$ \\
\hline $\mathrm{Zn}(1)-\mathrm{O}(2)$ & $2.022(5)$ & $\mathrm{Zn}(3)-\mathrm{N}(5)$ & $2.104(6)$ \\
\hline $\mathrm{Zn}(1)-\mathrm{N}(2)$ & $2.135(6)$ & $\mathrm{Zn}(3)-\mathrm{N}(6)$ & $2.122(7)$ \\
\hline $\mathrm{Zn}(1)-\mathrm{O}(1) \# 1$ & $2.184(5)$ & $\mathrm{Zn}(4)-\mathrm{O}(8)$ & $2.010(5)$ \\
\hline $\mathrm{Zn}(1)-\mathrm{N}(1)$ & $2.196(6)$ & $\mathrm{Zn}(4)-\mathrm{O}(7)$ & $2.038(5)$ \\
\hline $\mathrm{Zn}(1)-\mathrm{O}(4)$ & $2.262(5)$ & $\mathrm{Zn}(4)-\mathrm{N}(8)$ & $2.133(6)$ \\
\hline $\mathrm{Zn}(2)-\mathrm{O}(3)$ & $1.980(5)$ & $\mathrm{Zn}(4)-\mathrm{O}(8) \# 2$ & $2.170(5)$ \\
\hline $\mathrm{Zn}(2)-\mathrm{O}(2)$ & $2.008(5)$ & $\mathrm{Zn}(4)-\mathrm{N}(7)$ & $2.172(6)$ \\
\hline $\mathrm{Zn}(2)-\mathrm{O}(4)$ & $2.051(5)$ & $\mathrm{Zn}(4)-\mathrm{O}(6)$ & $2.277(5)$ \\
\hline $\mathrm{Zn}(2)-\mathrm{N}(4)$ & $2.095(7)$ & $\mathrm{O}(1)-\mathrm{Zn}(1) \# 1$ & $2.184(5)$ \\
\hline $\mathrm{Zn}(2)-\mathrm{N}(3)$ & $2.098(6)$ & $\mathrm{Zn}(3)-\mathrm{O}(5)$ & $1.975(5)$ \\
\hline $\mathrm{O}(1)-\mathrm{Zn}(1)-\mathrm{O}(2)$ & $170.8(2)$ & $\mathrm{O}(5)-\mathrm{Zn}(3)-\mathrm{O}(6)$ & $172.4(2)$ \\
\hline $\mathrm{O}(1)-\mathrm{Zn}(1)-\mathrm{N}(2)$ & $97.4(2)$ & $\mathrm{O}(7)-\mathrm{Zn}(3)-\mathrm{O}(6)$ & $82.75(19)$ \\
\hline $\mathrm{O}(2)-\mathrm{Zn}(1)-\mathrm{N}(2)$ & $91.5(2)$ & $\mathrm{O}(5)-\mathrm{Zn}(3)-\mathrm{N}(5)$ & $88.1(2)$ \\
\hline $\mathrm{O}(1)-\mathrm{Zn}(1)-\mathrm{O}(1) \# 1$ & $79.9(2)$ & $\mathrm{O}(7)-\mathrm{Zn}(3)-\mathrm{N}(5)$ & $104.8(2)$ \\
\hline $\mathrm{O}(2)-\mathrm{Zn}(1)-\mathrm{O}(1) \# 1$ & $101.9(2)$ & $\mathrm{O}(6)-\mathrm{Zn}(3)-\mathrm{N}(5)$ & $87.3(2)$ \\
\hline $\mathrm{N}(2)-\mathrm{Zn}(1)-\mathrm{O}(1) \# 1$ & $92.5(2)$ & $\mathrm{O}(6)-\mathrm{Zn}(3)-\mathrm{N}(5)$ & $87.3(2)$ \\
\hline $\mathrm{O}(1)-\mathrm{Zn}(1)-\mathrm{N}(1)$ & $86.9(2)$ & $\mathrm{O}(5)-\mathrm{Zn}(3)-\mathrm{N}(6)$ & $87.6(3)$ \\
\hline $\mathrm{O}(2)-\mathrm{Zn}(1)-\mathrm{N}(1)$ & $84.1(2)$ & $\mathrm{O}(7)-\mathrm{Zn}(3)-\mathrm{N}(6)$ & $114.8(2)$ \\
\hline $\mathrm{N}(2)-\mathrm{Zn}(1)-\mathrm{N}(1)$ & $173.9(2)$ & $\mathrm{O}(6)-\mathrm{Zn}(3)-\mathrm{N}(6)$ & $92.0(2)$ \\
\hline $\mathrm{O}(1) \# 1-\mathrm{Zn}(1)-\mathrm{N}(1)$ & $92.5(2)$ & $\mathrm{N}(5)-\mathrm{Zn}(3)-\mathrm{N}(6)$ & $140.1(3)$ \\
\hline $\mathrm{O}(1)-\mathrm{Zn}(1)-\mathrm{O}(4)$ & $101.9(2)$ & $\mathrm{O}(8)-\mathrm{Zn}(4)-\mathrm{O}(7)$ & $171.8(2)$ \\
\hline $\mathrm{O}(2)-\mathrm{Zn}(1)-\mathrm{O}(4)$ & $76.7(2)$ & $\mathrm{O}(8)-\mathrm{Zn}(4)-\mathrm{N}(8)$ & $95.7(2)$ \\
\hline $\mathrm{N}(2)-\mathrm{Zn}(1)-\mathrm{O}(4)$ & $84.8(2)$ & $\mathrm{O}(7)-\mathrm{Zn}(4)-\mathrm{N}(8)$ & $92.1(2)$ \\
\hline $\mathrm{O}(1) \# 1-\mathrm{Zn}(1)-\mathrm{O}(4)$ & $176.9(2)$ & $\mathrm{O}(8)-\mathrm{Zn}(4)-\mathrm{O}(8) \# 2$ & $79.7(2)$ \\
\hline
\end{tabular}




\begin{tabular}{llll}
\hline $\mathrm{N}(1)-\mathrm{Zn}(1)-\mathrm{O}(4)$ & $90.1(2)$ & $\mathrm{O}(7)-\mathrm{Zn}(4)-\mathrm{O}(8) \# 2$ & $102.6(2)$ \\
$\mathrm{O}(3)-\mathrm{Zn}(2)-\mathrm{O}(2)$ & $100.5(2)$ & $\mathrm{N}(8)-\mathrm{Zn}(4)-\mathrm{O}(8) \# 2$ & $92.8(2)$ \\
$\mathrm{O}(3)-\mathrm{Zn}(2)-\mathrm{O}(4)$ & $176.2(2)$ & $\mathrm{O}(8)-\mathrm{Zn}(4)-\mathrm{N}(7)$ & $86.7(2)$ \\
$\mathrm{O}(2)-\mathrm{Zn}(2)-\mathrm{O}(4)$ & $82.0(2)$ & $\mathrm{O}(7)-\mathrm{Zn}(4)-\mathrm{N}(7)$ & $85.3(2)$ \\
$\mathrm{O}(3)-\mathrm{Zn}(2)-\mathrm{N}(4)$ & $88.9(2)$ & $\mathrm{N}(8)-\mathrm{Zn}(4)-\mathrm{N}(7)$ & $174.2(2)$ \\
$\mathrm{O}(2)-\mathrm{Zn}(2)-\mathrm{N}(4)$ & $114.2(2)$ & $\mathrm{O}(8) \# 2-\mathrm{Zn}(4)-\mathrm{N}(7)$ & $92.8(2)$ \\
$\mathrm{O}(4)-\mathrm{Zn}(2)-\mathrm{N}(4)$ & $92.6(2)$ & $\mathrm{O}(8)-\mathrm{Zn}(4)-\mathrm{O}(6)$ & $101.7(2)$ \\
$\mathrm{O}(3)-\mathrm{Zn}(2)-\mathrm{N}(3)$ & $89.4(2)$ & $\mathrm{O}(7)-\mathrm{Zn}(4)-\mathrm{O}(6)$ & $76.5(2)$ \\
$\mathrm{O}(2)-\mathrm{Zn}(2)-\mathrm{N}(3)$ & $107.4(2)$ & $\mathrm{N}(8)-\mathrm{Zn}(4)-\mathrm{O}(6)$ & $83.9(2)$ \\
$\mathrm{O}(4)-\mathrm{Zn}(2)-\mathrm{N}(3)$ & $87.1(2)$ & $\mathrm{O}(8) \# 2-\mathrm{Zn}(4)-\mathrm{O}(6)$ & $176.5(2)$ \\
$\mathrm{N}(4)-\mathrm{Zn}(2)-\mathrm{N}(3)$ & $137.9(3)$ & $\mathrm{N}(7)-\mathrm{Zn}(4)-\mathrm{O}(6)$ & $90.5(2)$ \\
$\mathrm{O}(5)-\mathrm{Zn}(3)-\mathrm{O}(7)$ & $104.3(2)$ & $\mathrm{Zn}(1)-\mathrm{O}(1)-\mathrm{Zn}(1) \# 1$ & $100.1(2)$ \\
\hline
\end{tabular}

